\title{
Cardiovascular testing in patients with postural orthostatic tachycardia syndrome and Ehlers-Danlos syndrome type III
}

\author{
Svetlana Blitshteyn ${ }^{1} \cdot$ David Fries $^{2}$
}

Received: 2 January 2017/Accepted: 5 January 2017/Published online: 18 January 2017

(C) Springer-Verlag Berlin Heidelberg 2017

Keywords Postural orthostatic tachycardia syndrome . Ehlers-Danlos syndrome type III · Cardiovascular testing · Applanation tonometry

We read with interest the study by Cheng et al. that found no difference in the results of the 2D cardiac echocardiography and applanation tonometry between nine patients with postural orthostatic tachycardia syndrome (POTS) and Ehlers-Danlos syndrome (EDS) type III and age-matched controls [1]. We disagree with the authors' conclusion that their study comprehensively assessed the cardiovascular profile of patients with POTS and EDS type III. First, the tests were performed in the supine position, despite orthostatic intolerance being the hallmark of POTS. Second, arterial wall stiffness is a marker of arteriosclerosis and atherosclerosis, none of which are prevalent in the predominantly young POTS/EDS type III population. Third, to our knowledge, the utility of the applanation tonometry in connective tissue disorders, such as EDS type III, has not been studied, and as such, the efficacy of this method in assessing arterial wall compliance in EDS/POTS is unknown. Fourth, invasive cardiopulmonary exercise testing of patients with POTS documented low ventricular filling pressures as a cause of exercise intolerance vs. high

This is a letter commenting on J. L. Cheng et al. Clin Auton Res 2016.

Svetlana Blitshteyn

sb25@buffalo.edu

1 Dysautonomia Clinic, Department of Neurology, University at Buffalo School of Medicine and Biomedical Sciences, 100 High Street, Buffalo, NY 14203, USA

2 Division of Cardiovascular Disease, Sands-Constellation Heart Institute, Rochester General Hospital, Rochester, NY, USA filling pressures in deconditioned patients [2, 3]. Lastly, adrenergic antibodies in patients with POTS should be considered as a possible alternative explanation for the orthostatic intolerance $[4,5]$.

Compliance with ethical standards

Funding None.

Conflict of interest None.

\section{References}

1. Cheng JL, Au JS, Guzman JC et al (2016) Cardiovascular profile in postural orthostatic tachycardia syndrome and Ehlers-Danlos syndrome type III. Clin Auton Res. doi:10.1007/s10286-0160392-4

2. Oldham WM, Lewis GD, Opotowsky AR, Waxman AB, Systrom DM (2016) Unexplained exertional dyspnea caused by low ventricular filling pressures: results from clinical invasive cardiopulmonary exercise testing. Pulm Circ 6:55-62

3. Blitshteyn S, Fries D (2016) Postural tachycardia syndrome is not caused by deconditioning. Pulm Circ 6:401

4. Li H, Yu X, Liles C et al (2014) Autoimmune basis for postural tachycardia syndrome. J Am Heart Assoc 3:e000755

5. Fedorowski A, Li H, Yu X, et al. (2016) Antiadrenergic autoimmunity in postural tachycardia syndrome. Europace. doi:10.1093/europace/euw154 